\title{
Caracterización de la distribución del tamaño de los adipocitos para el estudio del tejido adiposo en producción animal
}

\author{
L. Alfonso* y J.A. Mendizabal
}

Escuela Técnica Superior de Ingenieros Agrónomos, Universidad Pública de Navarra, Campus Arrosadia, Pamplona, España

\section{Resumen}

El tamaño de los adipocitos, principales células constituyentes del tejido adiposo, ha sido objeto de numerosos estudios por el hecho de estar relacionado con el grado de desarrollo y la actividad metabólica de ese tejido. Su análisis resulta en ocasiones complejo dada la bimodalidad de su distribución. Este trabajo presenta un método de análisis basado en contrastar, en primer lugar la unimodalidad de la distribución del tamaño de los adipocitos, frente a una distribución bimodal. Posteriormente propone algunos parámetros para describir adecuadamente la distribución bimodal. Para su aplicación se desarrolló un sencillo programa informático que se utiliza, a modo de ejemplo, para analizar los datos de dos trabajos, previamente publicados, sobre el desarrollo adipocitario. Los resultados muestran el interés de utilizar contrastes de bimodalidad frente a la mera inspección visual de los histogramas de distribución. Además, cuando la hipótesis de unimodalidad es rechazada, la utilización de parámetros descriptivos de bimodalidad, como el porcentaje de adipocitos por encima del punto de inflexión entre ambas modas, permite una comparación más adecuada entre tratamientos experimentales.

Palabras clave: Célula grasa, hiperplasia, hipertrofia, bimodalidad, contraste.

\begin{abstract}
Characterizing adipocyte size distribution for adipose tissue studies in animal production

The size of the adipocytes, the main cells of the adipose tissue, has been the objective of several studies because of its relationship with the development and metabolic activity of this tissue. The bimodality of their distribution into the tissue makes the analysis sometimes difficult. Thus, a method based, on testing the unimodal distribution vs. bimodality is presented. Some descriptive parameters appropriate for the bimodal distribution are also shown. A simple software has been developed for its application, being used to analyse data from two previously published studies about adipocyte development. Results show the interest in the use of the test of bimodality rather than the simple visual inspection of distribution histograms. Furthermore, when unimodality is rejected, the use of descriptive parameters of bimodality, as the percentage of adipocytes above the inflection point between the two peaks, allows a better comparison among experimental treatments.
\end{abstract}

Key words: Adipose cell, hyperplasia, hypertrophy, bimodality, testing.

\footnotetext{
* Autor para correspondencia: leo.alfonso@unavarra.es http://dx.doi.org/10.12706/itea.2016.010
} 


\section{Introducción}

El conocimiento del tejido adiposo de los animales domésticos es de gran relevancia en producción animal dadas las implicaciones que tiene sobre el desarrollo y crecimiento de los animales, los resultados reproductivos, la calidad sensorial y nutricional de los productos y la eficiencia en la utilización de los recursos alimentarios, entre otros. Por ello, tanto el estudio de su formación (adipogénesis) como de su metabolismo (lipogénesis y lipólisis) son de gran importancia y han sido objeto de numerosos trabajos (Dodson et al., 2010), algunos de ellos relativos a nuestras poblaciones animales y sistemas de producción (Alzón et al., 2007; Soret et al., 1998).

El tejido adiposo presenta una elevada plasticidad y mantiene la capacidad de crecer a lo largo de toda la vida de los animales a través de procesos de hiperplasia (aumento del número de células) e hipertrofia (aumento del volumen celular). El estudio de ambos procesos precisa una adecuada caracterización de las principales células constituyentes del tejido adiposo, los adipocitos. En principio se piensa que la hipertrofia es el proceso que fundamentalmente contribuye al aumento de la cantidad de tejido adiposo, y que la hiperplasia ocurre únicamente en estados tempranos de desarrollo (Jo et al., 2009). Sin embargo no se puede descartar que ambos mecanismos se puedan producir simultáneamente, entre otras razones debido a que a medida que aumenta el tamaño de los adipocitos aumenta la hipoxia local produciendo su propia muerte, pero también la secreción, a partir de cierto volumen crítico, de factores de reclutamiento de nuevos adipocitos (Sun et al., 2011).

Como consecuencia de esa relación de los procesos de hiperplasia e hipertrofia celular pueden convivir distintas poblaciones de adipocitos maduros en distinto grado de expansión celular. Por otra parte, se puede pensar que la actividad metabólica de los adipocitos depende, de algún modo, de la magnitud de su propia superficie celular, dado que el intercambio de triglicéridos con el medio celular se produce a través de esta. Por lo que, diferencias en el flujo lipolítico y lipogénico podrían también dar lugar a distintas poblaciones de adipocitos de acuerdo a su tamaño sin que necesariamente existiesen procesos de hiperplasia celular (Soula et al., 2013).

De cualquier forma, sea como consecuencia de mecanismos adipogénicos o lipogénicos, o ambos conjuntamente, desde hace ya tiempo se han descrito distribuciones del tamaño de los adipocitos en especies animales que no se ajustan a la distribución normal y se pueden considerar bimodales (Hood y Allen, 1973; DeMartinis y Francendese, 1982). La existencia de bimodalidad en la distribución de los datos dificulta el análisis estadístico de los mismos. Para llevarlo a cabo de forma apropiada se ha pensado en la utilización de la distribución lognormal (Whitehurst et al., 1981), la mezcla de dos distribuciones normales (Mersmann et al., 1975) o incluso la mezcla de dos distribuciones exponenciales y una normal (McLaughlin et al., 2007). Sin embargo, no siempre es evidente cuándo considerar que los datos presentan una distribución bimodal, pues los métodos exploratorios, como por ejemplo la utilización de histogramas, pueden resultar engañosos (Fisher et al., 1994). Cuantificar si la distribución de un conjunto de datos se describe mejor con una o dos modas no es un problema de fácil solución (Muratov y Gnedin, 2010).

Tampoco resulta evidente cómo estudiar el tamaño de los adipocitos, en trabajos enfocados a contrastar diferencias en el desarrollo del tejido adiposo debidas a factores productivos como el genotipo, la edad, el sexo, la dieta, etc., cuando los datos del tamaño de los adipocitos presentan una distribución bimodal. Así, por ejemplo, Mendizabal et al. (2004), trabajando con corderos cebados intensivamente, optaron por agrupar los adi- 
pocitos en distintas clases según tamaño, y comparar las frecuencias de adipocitos según su tamaño sin hacer asunciones sobre su distribución. Cruz et al. (2012), por su parte, propusieron considerar la mezcla de dos distribuciones normales para explicar la bimodalidad de la distribución del tamaño de los adipocitos que observaron en vacuno de carne, y hacer las comparaciones entre grupos de animales a través de las diferencias en las medias y las varianzas de ambas distribuciones normales. McLaughlin et al. (2007) trabajando en estudios de obesidad en humanos, propusieron estimar parámetros propios de la distribución bimodal como las dos modas, el punto de inflexión menor (nadir) o el porcentaje de adipocitos sobre el nadir, para hacer comparaciones. Este último enfoque, se ha considerado que informa adecuadamente sobre los mecanismos de deposición de grasa y su relación con las enfermedades vinculadas a la obesidad (Jo et al., 2012), por lo que está siendo bastante utilizado en ese área de trabajo (Eliasson et al., 2014; Hadji et al., 2014; Soula et al., 2015).

El objetivo de este trabajo es presentar una metodología de análisis del tamaño de los adipocitos, sustentada en un programa informático elaborado para dicho fin, que persigue describir la unimodalidad o bimodalidad de la distribución de los adipocitos, y calcular parámetros adecuados de comparación entre grupos de datos. Para ilustrarla, se desarrolla un ejemplo a partir de los datos procedentes de dos trabajos, previamente publicados, sobre el desarrollo adipocitario.

\section{Material y métodos}

Análisis de la distribución del tamaño de los adipocitos

El programa desarrollado para analizar la distribución del tamaño de los adipocitos se basa en contrastar en primer lugar la unimodalidad de los datos, y en segundo lugar analizarlos utilizando distintos estadísticos en función de que la hipótesis de unimodalidad sea o no rechazada.

Partiendo del diámetro de los adipocitos como medida de su tamaño, el programa realiza los siguientes análisis. Primero calcula el histograma del tamaño de los adipocitos considerando como número de clases (nc) el entero de la raíz cuadrada del número de datos (adipocitos medidos) más uno, estableciendo un valor mínimo de 6 clases y un valor máximo de 20. Como amplitud de cada clase se toma el rango de los datos (valor máximo menos valor mínimo) dividido por el número de clases (rango/nc). Los histogramas obtenidos se normalizan dividiendo la frecuencia de cada clase por su amplitud para que su área corresponda siempre a la unidad y así hacerlos comparables entre grupos de datos (animales, tratamientos, tejidos, etc).

La utilización de estos histogramas como método exploratorio puede ofrecer una primera información sobre la distribución del diámetro de los adipocitos, pero a veces puede resultar engañosa (Fisher et al., 1994). Por ello, el análisis de los datos se prosigue estableciendo y contrastando la hipótesis nula de unimodalidad. El programa utiliza para ello el Coeficiente de Bimodalidad ( $B C$ ) (SAS Institute Inc, 1989) y el estadístico Dip (Hartigan y Hartigan, 1985), que se explican a continuación.

El Coeficiente de Bimodalidad es:

$$
B C=\frac{m_{3}^{2}+1}{\left(m_{4}+3 \frac{(n-1)^{2}}{(n-2)(n-3)}\right)}
$$

donde $\mathrm{n}$ es el número de observaciones, $\mathrm{m}_{3}$ es el sesgo y $\mathrm{m}_{4}$ el exceso de curtosis o apuntamiento.

Como se deduce de esa expresión, el cálculo de $B C$ es sencillo, pues se basa únicamente 
en el número de observaciones, y el sesgo y el exceso de curtosis de la distribución, aunque la existencia de distintas fórmulas para calcular sesgo y curtosis puede inducir confusión (Knapp, 2007; Pfister et al., 2013). Las expresiones utilizadas son las siguientes:

$$
\begin{gathered}
m_{3}=n \frac{\sum\left(\frac{x_{i}-\mu_{x}}{\sigma_{x}}\right)^{3}}{(n-1)(n-2)} \\
m_{4}=\left(n \frac{(n+1)}{(n-1)(n-2)(n-3)}\right)\left(\frac{\sum\left(x_{i}-\mu_{x}\right)^{4}}{\left(\sigma_{x}^{2}\right)^{2}}\right)-\left(3 \frac{(n-1)^{2}}{(n-2)(n-3)}\right)
\end{gathered}
$$

Para una distribución uniforme el valor de $B C$ es 0,555 , por lo que valores mayores pueden indicar ausencia de unimodalidad, aunque es cierto que también podrían estar indicando distribuciones unimodales fuertemente sesgadas.

El estadístico Dip es la máxima diferencia entre la función de distribución empírica, y la función de distribución unimodal que minimiza esa máxima diferencia (Hartigan, 1985). El contraste de Dip parte de la hipótesis nula de unimodalidad en la distribución de los datos, y aunque existen pruebas más potentes para contrastar la bimodalidad, precisan la asunción previa de alguna distribución concreta (Muratov y Gnedin, 2010). A diferencia de $B C$, el cálculo de Dip no está implementado en los paquetes estadísticos comerciales más habituales. En este trabajo, para su cálculo se utilizó la subrutina "Diptst" (Hartigan, 1985; modificada por Mechler, 2002), que también calcula el intervalo modal, que corresponde a los valores $a$ y $b$ de la distribución uniforme $(f(x)=0$ para $x<a$; $f(x)=$ $f(x)=\frac{1}{(b-a)}$ para $\left.a \leq x \leq b\right) ; f(x)=0$ para $x>b$ ) de menor diferencia con la distribución empírica.

La significación de la hipótesis de distribución unimodal se evalúa comparando el valor del estadístico Dip con los valores empíricos obtenidos bajo esa hipótesis para un nivel de significación del $5 \%$, a partir del paquete "diptest" de R (Maechler, 2013) y posterior in- terpolación basada en $\sqrt{ } n$ Dip (Hartigan, 1985; siendo $n$ el número de observaciones). Si el valor de Dip es menor que el valor correspondiente a la significación del $5 \%$ no se rechaza la hipótesis nula de unimodalidad, y dado el carácter conservador del test, el programa calcula también el valor correspondiente a una significación del $10 \%$ para valorar mejor la posible existencia de bimodalidad.

Si ambas pruebas, BC y Dip, rechazan la hipótesis de unimodalidad, se puede considerar que los datos se distribuyen bimodalmente. Cuando los resultados de ambas pruebas no convergen, se puede pensar en analizar otros criterios del tamaño de los adipocitos (como superficie o volumen) y, en última instancia, considerar la prueba Dip como una medida más adecuada de la bimodalidad que el $B C$ (Freeman y Dale, 2013).

Una vez contrastada la hipótesis de unimodalidad, se calculan los estadísticos habituales de una población que sigue una distribución normal (media, mediana, moda, varianza, mínimo, máximo, coeficientes de asimetría y apuntamiento, etc.) y algunos parámetros descriptivos de las distribuciones bimodales como ambas modas, el punto de inflexión entre ambas (nadir), el porcentaje de adipocitos sobre el nadir y la relación entre el número de adipocitos pequeños (por debajo del nadir) y grandes (por encima) (McLaughlin et al., 2007). En función de que se rechace o no la unimodalidad de los datos, se deberán 
considerar los estadísticos apropiados en la comparación de distintos conjuntos de datos.

El cálculo de los parámetros descriptivos de la bimodalidad se realiza por aproximación y no por ajuste a distribuciones teóricas, dado que no se asume ninguna distribución subyacente bajo la distribución bimodal de los datos. La aproximación se basa en el histograma previamente calculado y consiste en asumir, partiendo de la primera clase, que una clase es la primera moda si su frecuencia es mayor a todas las anteriores y menor a $n$ clases posteriores. El valor de $n$ varía en función del número de clases del histograma, calculándose como el valor entero del número total de clases dividido por 10 (o, lo que es igual, de la raíz cuadrada del número de observaciones dividido por 10), dentro del intervalo $[1,5]$. Así hasta 224 observaciones, $n=1$; de 225 a 624, $n=2$; de 625 a 1224, $n=3$; de 1225 a 2024, $n=4$; y para más de 2025 observaciones, $n=5$. El cálculo del nadir (punto de inflexión de la distribución bimodal) se calcula asumiendo que la frecuencia de todas las clases a partir de la primera moda estimada debe de ser mayor, y las $n$ clases siguientes también mayores (usando el mismo valor de $n$ ). Finalmente, la segunda moda se estima de forma análoga a la primera pero partiendo del nadir previamente calculado, y considerando que es posible que sobre él haya más de una moda, por lo que se considera la mayor de todas ellas.

El programa desarrollado en Fortran (Fortran95 PRO,v5.7, Lahey Computer Systems, Inc.), está adaptado mediante Liberty Basic (version 4.04, Shoptalk Systems) al entorno MS-Windows. Incorpora la posibilidad de analizar también superficie (S) y volumen (V) como criterios del tamaño de los adipocitos, pero partiendo siempre del diámetro (D) como variable medida, y asumiendo esfericidad perfecta de los adipocitos.

$$
\left[S=4 \pi\left(\frac{D}{2}\right)^{2} ; V=\frac{4}{3} \pi\left(\frac{D}{2}\right)^{3}\right]
$$

\section{Ejemplo considerado}

Para ilustrar la metodología de trabajo expuesta, se desarrolla un ejemplo a partir de los datos procedentes de dos trabajos previamente publicados (Alfonso et al., 2005; Abadía et al., 2008), en los que se utilizó la técnica de determinación del diámetro de los adipocitos basada en observaciones microscópicas y su posterior medida mediante la técnica de análisis de imagen (empleada desde hace años por parte del grupo de investigación; Mendizabal et al., 1997).

En ambos trabajos se analizaba la existencia de diferencias en el tejido adiposo en porcino. En el trabajo de Alfonso et al. (2005) se analizaron las diferencias en el tamaño de los adipocitos de la capa más externa del tejido adiposo subcutáneo entre cerdos en crecimiento de dos poblaciones claramente diferenciadas por su capacidad de deposición de grasa, una población de cerdos Large White y otra de cerdos Pie Noir du Pays Basque (raza incorporada con posterioridad al Catálogo Oficial de Razas de Ganado en España con el nombre de Euskal Txerria). Se dispuso de los datos de 22 cerdos Large White y 18 cerdos Euskal Txerria, con similar número de adipocitos medidos por animal, $226 \pm 5$ y $222 \pm 10$ adipocitos ( \pm error típico), respectivamente.

En el trabajo de Abadía et al. (2008) se analizaron las diferencias en el tamaño de los adipocitos de cerdas reproductoras de líneas comerciales, con el objetivo de ver si existían diferencias relacionadas con el desarrollo de cada una de las capas que constituyen el tejido adiposo subcutáneo. Se dispuso de los datos de 16 cerdas reproductoras, con un número similar de adipocitos medidos por animal, $294 \pm 30,256 \pm 26$, y $239 \pm 24$, en las capas externa, intermedia e interna del tejido adiposo subcutáneo, respectivamente. 


\section{Resultados y discusión}

Distribución del diámetro de los adipocitos

En la Figura 1 se observa que la distribución del diámetro de los adipocitos en cerdos de engorde presenta un aspecto bastante parecido a la distribución normal, con una única moda cuyo valor es algo mayor para los cerdos Euskal Txerria que para los cerdos Large White. En cerdas reproductoras, por contra, la distribución no presenta el aspecto característico de la distribución normal (Figura 2), asemejándose más a una distribución bimodal con un punto de inflexión entre 40 y 60 $\mu \mathrm{m}$ de diámetro.

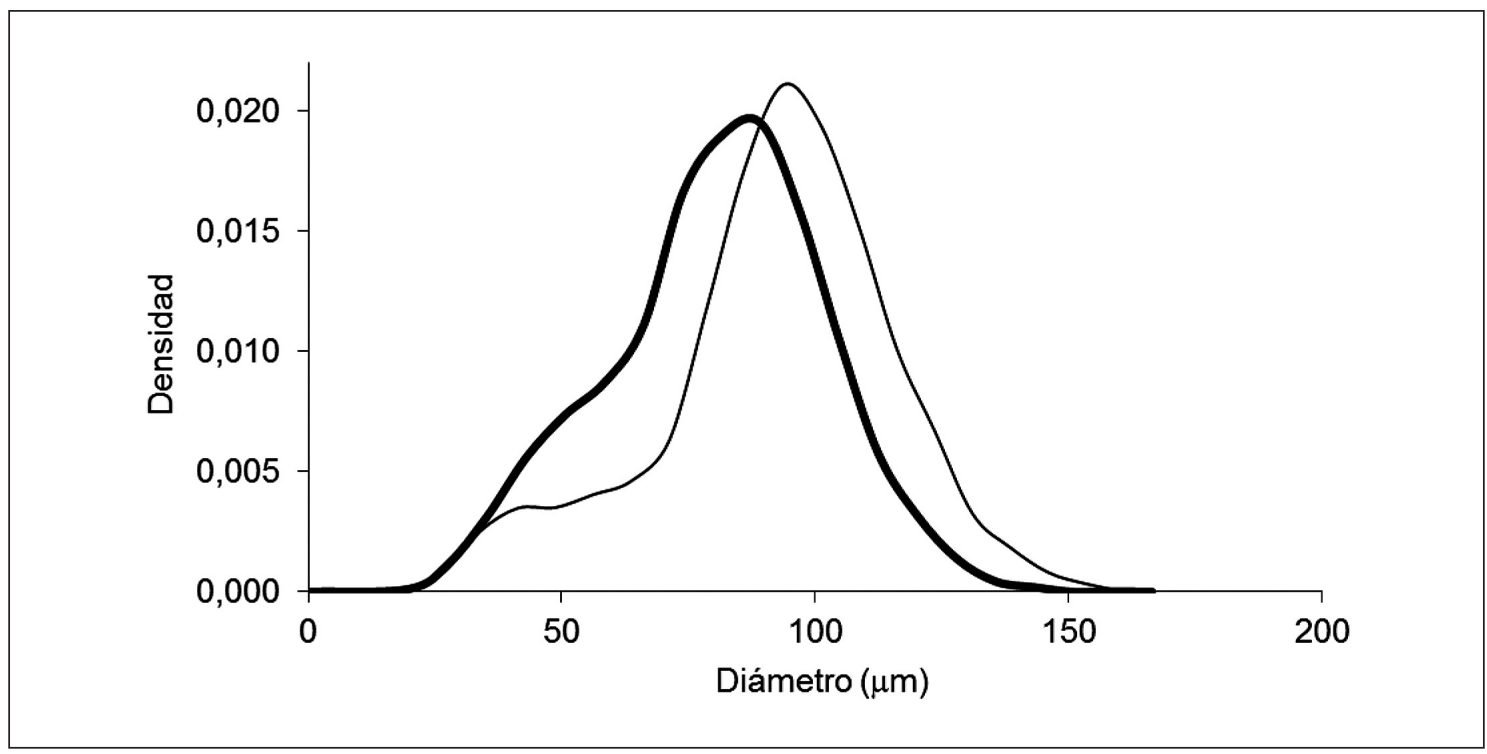

Figura 1. Distribución del diámetro de los adipocitos ${ }^{1}$ en la capa externa del tejido adiposo subcutáneo de cerdos Euskal Txerria (-) y Large White (-) en crecimiento ${ }^{2}$.

${ }^{1}$ Los resultados corresponden al análisis conjunto de los adipocitos medidos en todos los animales de cada grupo.

${ }^{2}$ Alfonso et al. (2005).

Figure 1. Adipocyte size distribution in outer subcutaneous backfat layer of Basque (-) and Large White (-) pigs during growing period.

Al contrastar la hipótesis nula de unimodalidad se obtiene que para cerdos en crecimiento no se puede rechazar la hipótesis nula de distribución unimodal de los adipocitos, que recordemos pertenecían a la capa externa del tejido adiposo subcutáneo (Tabla 1). Sin embargo, en cerdas reproductoras, la distribución del diámetro de los adipocitos de esa misma capa, la externa, se rechaza que sea unimodal. Mientras que en todos los casos se obtiene un coeficiente de asimetría negativo, indicando un aparente mayor número de adipocitos a la izquierda de la media (pequeños), para la capa externa en cerdas reproductoras es positivo. Para la capa interna también se rechaza la hipótesis de 


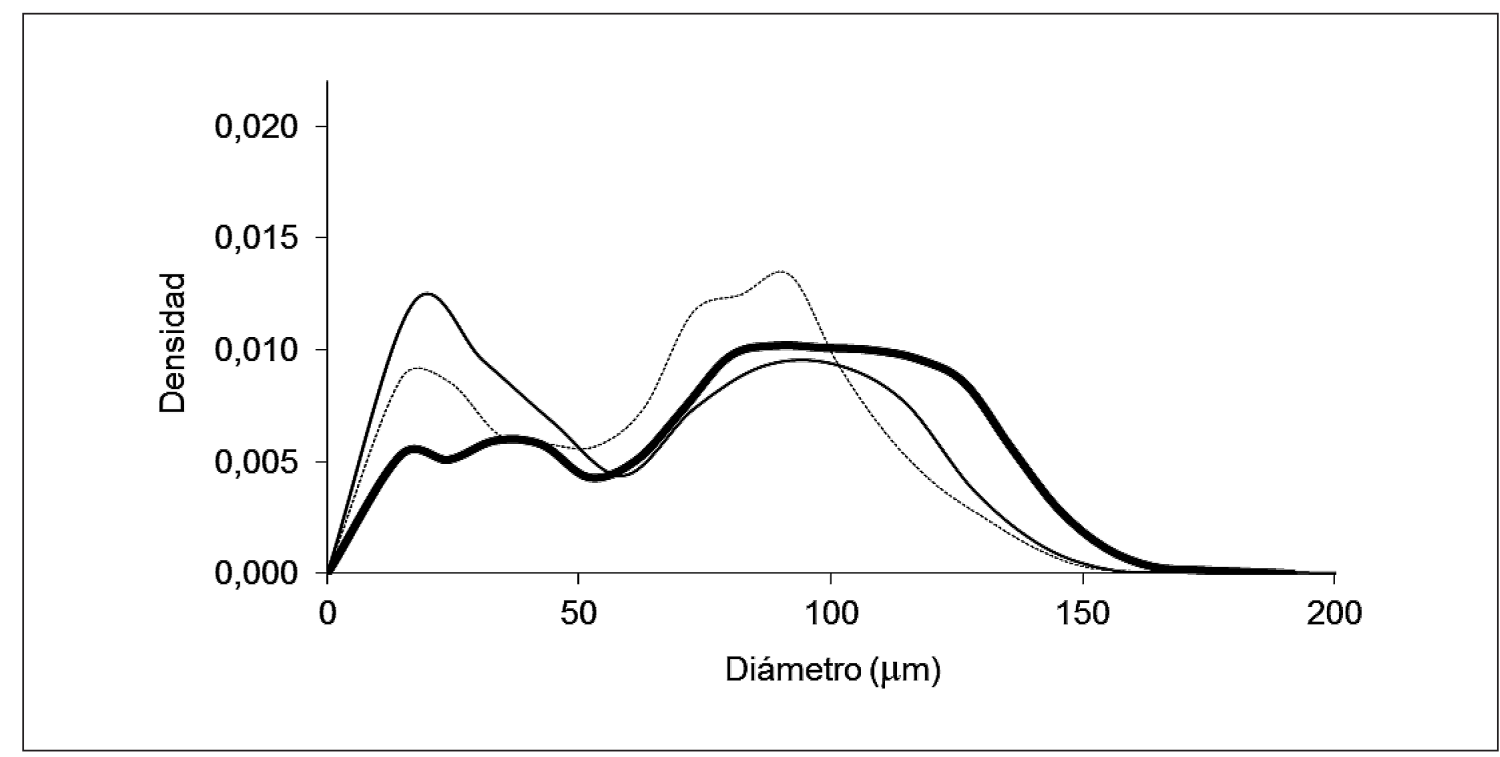

Figura 2. Distribución del diámetro de los adipocitos ${ }^{1}$ en las distintas capas que forman el tejido adiposo subcutáneo en cerdas reproductoras, externa (-), intermedia (-) e interna $(\cdots . .)^{2}$.

${ }^{1}$ Los resultados corresponden al análisis conjunto de los adipocitos medidos en todos los animales de cada grupo.

${ }^{2}$ Abadía et al. (2008).

Figure 2. Adipocyte size distribution in the individual layers of subcutaneous backfat tissue of sows, outer (-), middle (-) and inner (...) layers.

unimodalidad, pudiéndose mantener sólo en la capa intermedia la hipótesis de unimodalidad (Tabla 1). A ese resultado se llega atendiendo al estadístico Dip, dado que el Coeficiente de Bimodalidad, pese a ser mayor para cerdas reproductoras que para cerdos en cebo, no indica en ningún caso suficiente evidencia de bimodalidad.

El análisis de la distribución conjunta del tamaño de los adipocitos de todos los animales que conforman el grupo experimental puede, no obstante, enmascarar la existencia de importantes diferencias entre animales. Haciendo el análisis individualmente para cada animal, se observa cómo, en realidad, la distribución no es siempre del mismo tipo dentro de grupo de animales. Así, en la Figura 3 se puede ver que, como cabía esperar, existe una elevada variabilidad en la distribución del tamaño entre animales de un mismo grupo de comparación, especialmente en el grupo de cerdas reproductoras. Esta variabilidad puede implicar que para algunos animales se rechace la hipótesis de distribución unimodal mientras que para otros no. Para el grupo de cerdos Large White nunca se rechazó la unimodalidad. Para un $25 \%$ de los cerdos Euskal Txerria el $B C$ indicaba bimodalidad, aunque el contraste Dip no permitió rechazar la unimodalidad en ningún caso. En las cerdas reproductoras, la prueba $B C$ indicó existencia de bimodalidad en un $50 \%$, un $50 \%$ y un $44 \%$ de los animales para las capas externa, intermedia e interna respectivamente. Para la prueba Dip, los porcentajes 
(a)

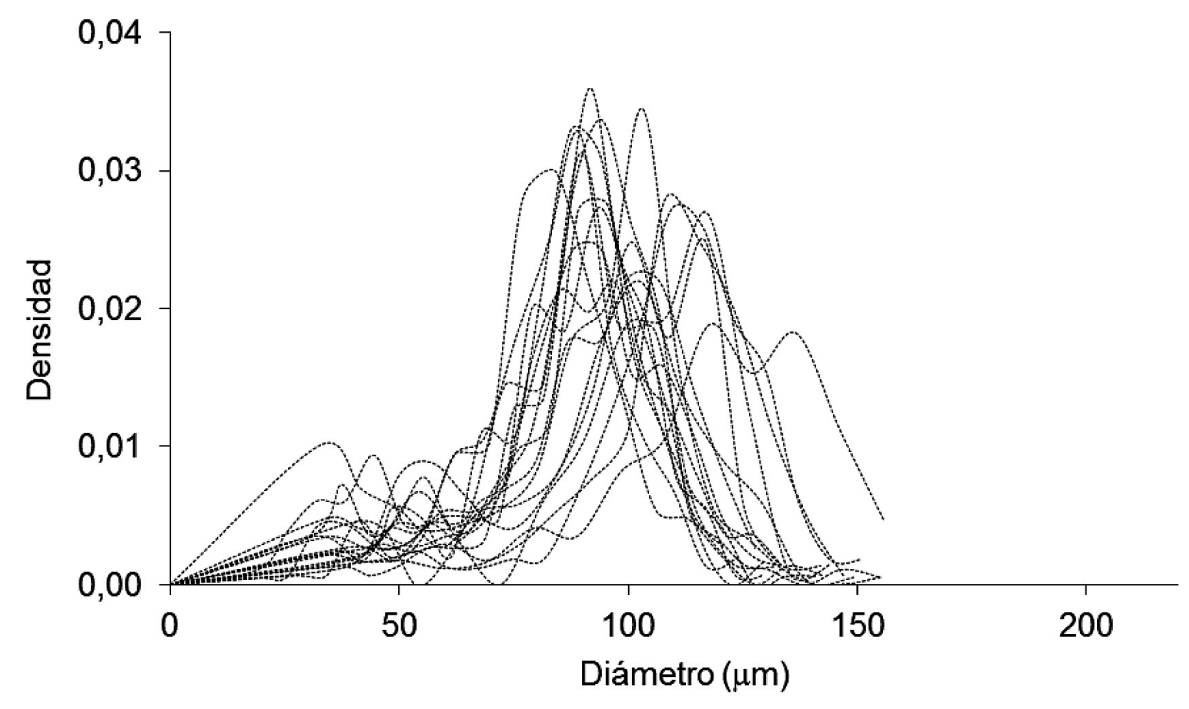

(b)

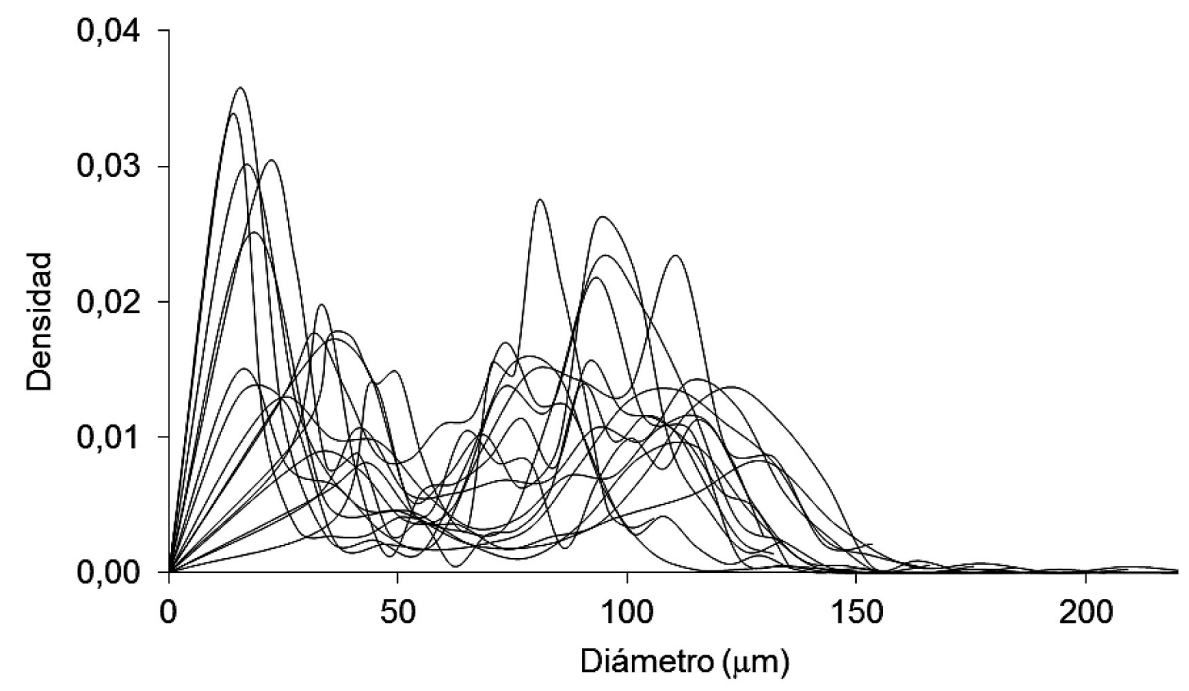

Figura 3. Distribución del diámetro de los adipocitos en la capa externa del tejido adiposo subcutáneo de (a) cerdos Euskal Txerria ${ }^{1}$, y (b) cerdas reproductoras comerciales ${ }^{2}$ al considerar la distribución en cada animal por separado.

${ }^{1}$ Alfonso et al. (2005); ${ }^{2}$ Abadía et al. (2008).

Figure 3. Adipocyte size distribution in the outer subcutaneous backfat layer of (a) Basque pigs, and (b) commercial sows when each animal was individually considered. 
Tabla 1. Resultados del contraste de la hipótesis de unimodalidad (H0) de las distribuciones del diámetro de los adipocitos medidos en el conjunto de los animales de cada grupo Table 1. Results of testing the unimodality hypothesis $(\mathrm{HO})$ of the adipocyte diameter measured jointly for animals from each group

\begin{tabular}{|c|c|c|c|c|c|}
\hline & \multicolumn{2}{|c|}{ Población ${ }^{4}$} & \multicolumn{3}{|c|}{$\mathrm{Capa}^{5}$} \\
\hline & Euskal Txerria & Large White & Externa & Intermedia & Interna \\
\hline \multicolumn{6}{|l|}{$B C^{1}$} \\
\hline Valor & 0,373 & 0,372 & 0,444 & 0,487 & 0,459 \\
\hline Curtosis & 0,322 & $-0,211$ & $-0,673$ & $-0,809$ & $-0,799$ \\
\hline Asimetría & $-0,489$ & $-0,196$ & 0,187 & $-0,261$ & $-0,109$ \\
\hline \multicolumn{6}{|l|}{ Inferencia ${ }^{2}$} \\
\hline Se rechaza $\mathrm{H} 0$ & No & No & No & No & No \\
\hline \multicolumn{6}{|l|}{$D I P^{3}$} \\
\hline Valor & 0,0058 & 0,0029 & 0,0324 & 0,0073 & 0,0184 \\
\hline \multicolumn{6}{|l|}{ Inferencia } \\
\hline $\operatorname{DIP}(\alpha=0,05)$ & $<0,0089$ & $<0,0074$ & $>0,0080$ & $<0,0080$ & $>0,0092$ \\
\hline $\operatorname{DIP}(\alpha=0,10)$ & $<0,0082$ & $<0,0069$ & & $<0,0081$ & \\
\hline Se rechaza $\mathrm{HO}$ & No & No & $\mathrm{Si}$ & No & $\mathrm{Si}$ \\
\hline
\end{tabular}

${ }^{1}$ Coeficiente de Bimodalidad.

2 Valores de $B C<0,555$ indican unimodalidad.

${ }^{3}$ Dip: estadístico que corresponde a la máxima diferencia entre la función de distribución empírica y la función de distribución unimodal que minimiza esa máxima diferencia.

${ }^{4}$ Alfonso et al. (2005).

${ }^{5}$ Abadía et al. (2008).

fueron del $75 \%, 44 \%$ y $31 \%$ para las capas externa, intermedia e interna, también respectivamente, que en este caso resultaron significativamente distintos al contrastarlos mediante una prueba $\chi^{2}(p=0,033)$.

Análisis de diferencias en la distribución del diámetro de los adipocitos entre grupos de animales

La comparación de la distribución del tamaño de los adipocitos entre los animales Large
White y Euskal Txerria es sencilla de realizar dado que, al no rechazar en ningún caso la distribución unimodal, podemos basarnos en los estadísticos habitualmente utilizados asumiendo normalidad. Los resultados se recogen en la Tabla 2, ratificando los publicados en Alfonso et al. (2005) que mostraban un mayor tamaño de los adipocitos de los animales Euskal Txerria.

La comparación de las distribuciones del tamaño de los adipocitos entre las distintas capas del tejido adiposo subcutáneo en cerdas 
Tabla 2. Valores medios ( \pm error típico) de los estadísticos descriptivos considerados en la comparación de la distribución del diámetro de los adipocitos en la capa externa del tejido adiposo subcutáneo de cerdos Large White y Euskal Txerria

Table 2. Mean values ( \pm standard error) of descriptive parameters considered for comparing the adipocyte size distribution of outer subcutaneous backfat layer in Basque and Large White pigs

\begin{tabular}{lcc}
\hline & Euskal Txerria & Large White \\
\hline Mínimo $(\mu \mathrm{m})$ & $30,66 \pm 1,70$ & $29,24 \pm 1,56$ \\
Máximo $(\mu \mathrm{m})$ & $139,23 \pm 2,61 \mathrm{a}$ & $128,33 \pm 2,87 \mathrm{~b}$ \\
Mediana $(\mu \mathrm{m})$ & $96,23 \pm 2,46 \mathrm{a}$ & $80,86 \pm 2,35 \mathrm{~b}$ \\
Media $(\mu \mathrm{m})$ & $92,55 \pm 2,32 \mathrm{a}$ & $79,71 \pm 2,20 \mathrm{~b}$ \\
Moda $(\mu \mathrm{m})$ & $99,53 \pm 2,50 \mathrm{a}$ & $80,07 \pm 3,62 \mathrm{~b}$ \\
\hline
\end{tabular}

En una fila, letras distintas indican diferencias significativas, $\alpha=0,05$.

reproductoras no es, por el contrario, tan sencilla. La convivencia de ambas distribuciones, uni y bimodales, dentro de un mismo grupo de animales hace la comparación entre grupos más difícil, pues no es evidente qué parámetros de comparación considerar y cómo calcular la significación de sus diferencias entre grupos. La solución propuesta consiste en hacer en estos casos una doble comparación entre grupos; una entre aquellos animales que presentan una distribución unimodal en ambos grupos, y otra entre los que presentan una distribución bimodal. De este modo, se puede calcular el error de estimación de los parámetros descriptivos apropiados para cada tipo de distribución y la significación de las diferencias entre los grupos. Los resultados se recogen en la Tabla 3.

Para el conjunto de todos los animales se puede observar un gradiente en el tamaño máximo de los adipocitos que va desde la capa externa $(172 \mu \mathrm{m})$ a la interna $(138 \mu \mathrm{m})$. Ese resultado no se relaciona con el valor de la mediana, que resultó ser mayor en la capa intermedia que en las otras dos, posiblemente ligado con el carácter unimodal de la distribución de la capa intermedia (Tabla 1).
Al comparar los resultados correspondientes a los animales en los que las distribuciones tenían carácter unimodal podemos concluir que las capas interna e intermedia presentan adipocitos de distinto tamaño, siendo en media mayores en la capa intermedia, y de mayor tamaño los más frecuentes (Tabla 3).

La comparación entre los resultados correspondientes a los animales en los que las distribuciones tenían carácter bimodal aporta nuevos datos sobre las diferencias entre capas. Por una parte se observa que no existen diferencias significativas en las dos modas ni en el punto de inflexión entre ambas. Este punto de inflexión que separa ambas distribuciones se encuentra siempre entre 50 y 60 $\mu \mathrm{m}$. La primera distribución presenta una moda que se sitúa algo por encima de $20 \mu \mathrm{m}$, y la segunda alrededor de los $100 \mu \mathrm{m}$. Podemos, por tanto, pensar que la mezcla de distribuciones es siempre la misma para las tres capas. A partir de ahí, las diferencias podrían proceder de variaciones en el porcentaje de adipocitos que se sitúan por encima del punto de inflexión o, dicho de otra manera, que pertenecen a la población de adipocitos "pequeños" o "grandes". Siguiendo con esta 
Tabla 3. Valores medios ( \pm error típico) de los estadísticos descriptivos considerados en la comparación de la distribución del diámetro de los adipocitos en la capas externa, intermedia e interna del tejido adiposo subcutáneo de cerdas reproductoras

Table 3. Mean values ( \pm standard error) of descriptive parameters considered for comparing the adipocyte size distribution in outer, middle and inner subcutaneous backfat layers of sows

\begin{tabular}{lccc}
\hline & Externa & Intermedia & Interna \\
\hline Total de animales & & & \\
$\mathrm{n}$ & 16 & 16 & 16 \\
Mínimo $(\mu \mathrm{m})$ & $19,66 \pm 2,07 \mathrm{a}$ & $24,16 \pm 2,97 \mathrm{a}$ & $25,93 \pm 3,00 \mathrm{a}$ \\
Máximo $(\mu \mathrm{m})$ & $171,28 \pm 11,02 \mathrm{a}$ & $158,74 \pm 4,54 \mathrm{a}$ & $138,39 \pm 5,84 \mathrm{~b}$ \\
Mediana $(\mu \mathrm{m})$ & $72,75 \pm 5,40 \mathrm{a}$ & $95,81 \pm 5,14 \mathrm{~b}$ & $73,53 \pm 6,74 \mathrm{a}$ \\
\hline Animales con distribución unimodal & & & \\
$\mathrm{n}$ & 4 & 9 & 11 \\
Media $(\mu \mathrm{m})$ & $85,47 \pm 2,22 \mathrm{ab}$ & $93,49 \pm 4,90 \mathrm{a}$ & $76,54 \pm 6,59 \mathrm{~b}$ \\
Moda $(\mu \mathrm{m})$ & $98,48 \pm 5,08 \mathrm{ab}$ & $105,92 \pm 5,73 \mathrm{a}$ & $82,05 \pm 7,78 \mathrm{~b}$ \\
\hline Animales con distribución bimodal & & & \\
$\mathrm{n}$ & 12 & 7 & 5 \\
Nadir $(\mu \mathrm{m})$ & $58,15 \pm 3,84 \mathrm{a}$ & $54,77 \pm 6,80 \mathrm{a}$ & $55,90 \pm 6,06 \mathrm{a}$ \\
\% sobre el nadir & $54,87 \pm 3,86 \mathrm{a}$ & $68,05 \pm 4,16 \mathrm{~b}$ & $56,10 \pm 9,03 \mathrm{ab}$ \\
Pequeños/grandes & $0,93 \pm 0,14 \mathrm{a}$ & $0,50 \pm 0,09 \mathrm{~b}$ & $0,96 \pm 0,28 \mathrm{a}$ \\
Primera moda $(\mu \mathrm{m})$ & $23,89 \pm 2,37 \mathrm{a}$ & $20,92 \pm 2,74 \mathrm{a}$ & $22,41 \pm 6,36 \mathrm{a}$ \\
Segunda moda $(\mu \mathrm{m})$ & $101,60 \pm 5,63 \mathrm{a}$ & $105,27 \pm 9,82 \mathrm{a}$ & $100,22 \pm 11,19 \mathrm{a}$ \\
\hline
\end{tabular}

En una fila, letras distintas indican diferencias significativas, $\alpha=0,05$ ).

idea, se observa que realmente existen diferencias significativas en el porcentaje de adipocitos "grandes" o "pequeños" entre capas. En la capa intermedia existe un mayor porcentaje de adipocitos por encima del punto de inflexión que separa ambas distribuciones, resultando un ratio menor del número de pequeños/grandes (Tabla 3).

Estos resultados no contradicen los previamente publicados asumiendo la existencia de una única moda en la distribución del tamaño de los adipocitos en cada una de las cerdas analizadas (Abadía et al., 2008) pero sí los complementan. En el trabajo de Abadía et al. (2008) se alcanzaba a concluir que el diámetro medio de los adipocitos de la capa intermedia $(89,0 \pm 0,5)$ era mayor $(p<0,05)$ que el de las capas externa $(76,6 \pm 0,5)$ e interna $(76,3 \pm 0,5)$. Analizarlos asumiendo que la distribución pueda ser en algunos casos bimodal permite profundizar en ese resultado viendo que lo que está ocurriendo es que existe un conjunto de cerdas para las que la distribución es unimodal y que en media presentan un mayor ta- 
maño de adipocitos, y otro conjunto de cerdas para las que existe una mayor proporción de adipocitos grandes en la capa intermedia que en las otras dos. Dado que se asume que el tamaño de los adipocitos es un indicador del estado metabólico (Jo et al., 2102), ambas situaciones podrían indicar una mayor actividad lipogénica en esta capa intermedia, con una mayor intensidad de la hipertrofia adipocitaria que las otras dos capas.

Esa idea se ve en cierto modo avalada al analizar la relación entre los parámetros de las distribuciones que presentan diferencias significativas entre capas con el espesor de cada una de ellas, tal como se realizó en Abadía et al. (2008). Los resultados de ese análisis (Tabla 4) indican que existe una relación positiva entre el espesor de la capa de grasa y el valor medio máximo del tamaño de los adipocitos en la capa externa y la intermedia, que sugiere que a medida que la capa es más gruesa se encuentran adipocitos de mayor tamaño. No se encuentra tal relación significativa en la capa interna. También se observa que a medida que aumenta el espesor de grasa de la capa intermedia, la mediana se desplaza a la derecha, lo que podría indicar un papel más activo en el almacenaje de triglicéridos que en las otras dos capas. Finalmente también cabe mencionar que en la capa interna se observa que el aumento de su espesor de grasa se acompaña de una reducción del porcentaje de adipocitos "grandes", lo que podría indicar que predominan procesos de hiperplasia, al contrario de lo que ocurre en las capas intermedia y ex-

Tabla 4. Coeficientes de regresión lineal estimados ( \pm error típico) entre el espesor de cada capa de grasa subcutánea y el valor medio de los parámetros descriptivos para los que se encontraron diferencias significativas entre capas en cerdas reproductoras

Table 4. Linear regression coefficients ( \pm standard error) between the depth of each backfat layer and the mean value of descriptive parameters that showed significant differences among layers, in sows

\begin{tabular}{lccc}
\hline & Externa & Intermedia & Interna \\
\hline Total de animales & 16 & & \\
$\mathrm{n}$ & $8,27 \pm 3,42 *$ & $1,76 \pm 0,52 * *$ & 16 \\
Máximo & $\mathrm{NS}$ & $2,20 \pm 0,54 * *$ & $\mathrm{NS}$ \\
Mediana & & & $\mathrm{NS}$ \\
\hline Animales con distribución unimodal & 4 & 9 & 11 \\
$\mathrm{n}$ & $\mathrm{NS}$ & $\mathrm{NS}$ & $\mathrm{NS}$ \\
Media & $10,22 \pm 1,11 *$ & $\mathrm{NS}$ & $\mathrm{NS}$ \\
Moda & & & \\
\hline Animales con distribución bimodal & 12 & 7 & 5 \\
$\mathrm{n}$ & $\mathrm{NS}$ & $\mathrm{NS}$ & $-7,10 \pm 2,21$ * \\
$\%$ sobre el nadir & $\mathrm{NS}$ & $\mathrm{NS}$ & $\mathrm{NS}$ \\
Pequeños/grandes & & & \\
\hline
\end{tabular}

Fuente: Abadía et al. (2008). 
terna. No obstante, en este último caso y pese a la significación de los resultados, el escaso número de animales no permite ir más allá de una mera especulación.

Finalmente, se puede ver que el análisis de la distribución del tamaño, como alternativa al tamaño medio, puede ofrecer más información sobre el por qué se pueden estar produciendo diferencias en el tipo de distribución entre animales. Los datos de Abadía et al. (2008) no son apropiados para responder a esa pregunta, entre otras razones por el escaso número de animales analizados, pero de todas formas podemos formularnos la pregunta del porqué algunas cerdas reproductoras presentan una distribución bimodal y otras unimodal. A modo de ejemplo especulativo, podríamos pensar que la prácticamente ausencia de bimodalidad en la distribución de los adipocitos de la capa externa en cerdos en crecimiento, y la diferente frecuencia de bimodalidad observada en cerdas adultas entre las distintas capas, podrían indicar una posible relación con la diferencia temporal existente en el desarrollo de las tres capas, primero la externa, luego la intermedia y finalmente la interna (Alfonso, 2004); así, se podría especular que la frecuencia de la bimodalidad puede aumentar con la edad y el distinto grado de desarrollo de las capas asociado a esta.

Aunque los dos ejemplos desarrollados corresponden a la especie porcina, la forma de análisis propuesta es igualmente aplicable a otras especies, tal como se puede ver en la Figura 4 que recoge los resultados de reanalizar los datos de Mendizabal et al. (2004) correspondientes a adipocitos de corderos de raza Lacaune agrupados en tres categorías de acuerdo al grado de consistencia del tejido graso subcutáneo.

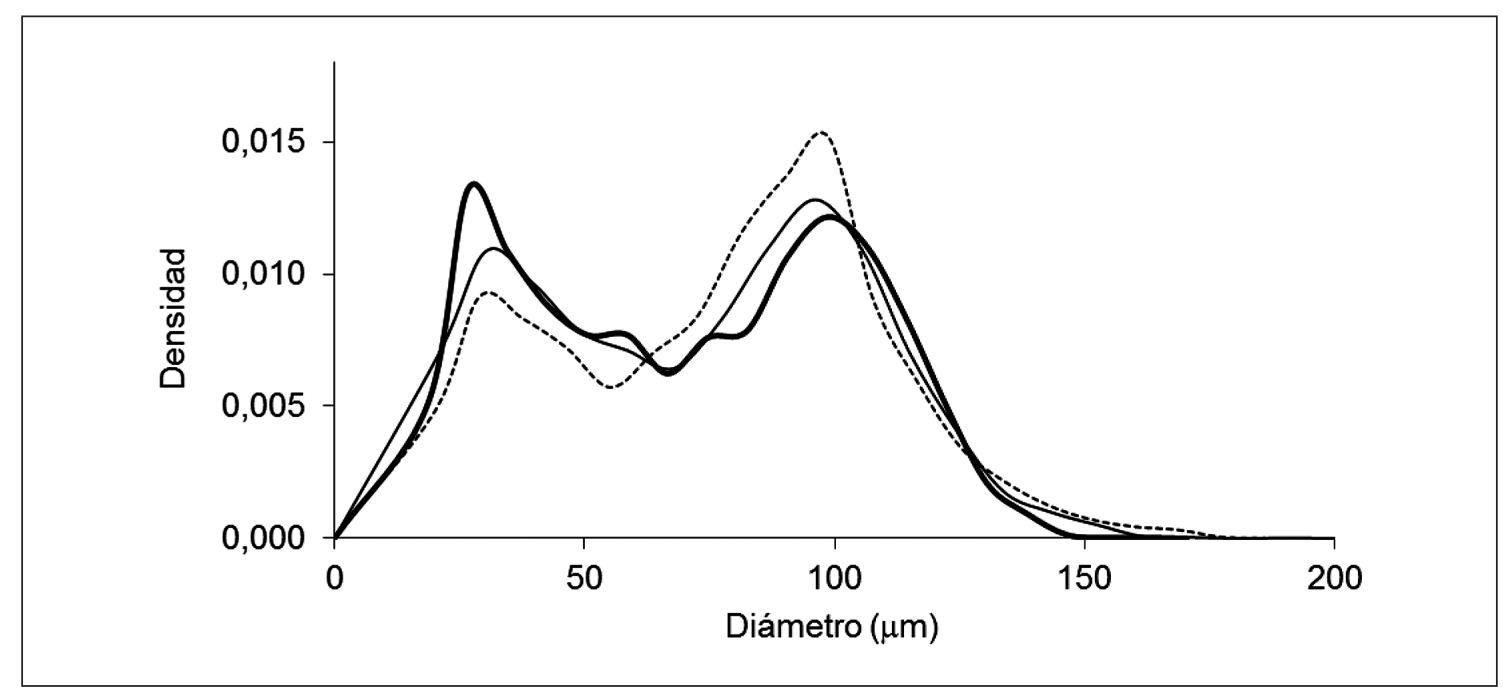

Figura 4. Distribución del diámetro de los adipocitos ${ }^{1}$ del tejido adiposo subcutáneo de corderos de raza Lacaune ${ }^{2}$.

${ }^{1}$ Los resultados corresponden al análisis conjunto de los adipocitos medidos en todos los animales de cada uno de los grupos que se consideraron de acuerdo a la consistencia de la grasa

(1: dura o muy dura (....); 2: medianamente blanda (-); 3: blanda o muy blanda (-)).

${ }^{2}$ Mendizabal et al. (2004).

Figure 4. Adipocyte size distribution in subcutaneous backfat tissue of Lacaune lambs. 


\section{Conclusiones}

Dado que el tamaño de los adipocitos no siempre se distribuye siguiendo una única distribución normal, unimodal, antes de hacer comparaciones en base a la media aritmética se recomienda investigar la posible existencia de bimodalidad. Para rechazar la hipótesis de unimodalidad, no es suficiente la inspección del histograma de frecuencias de la distribución, si no que es necesario realizar algún contraste estadístico adicional. De los dos utilizados en este trabajo, el Coeficiente de Bimodalidad y el estadístico Dip, el primero no parece aportar mucha información, por lo que se ratifica el Dip como más apropiado.

Cuando la hipótesis de unimodalidad se rechaza, la comparación de parámetros como el porcentaje de adipocitos por encima del punto de inflexión (nadir) de la distribución, es más apropiada que la comparación entre los valores de la media aritmética. En el caso en el que no todos los animales de un grupo de comparación presenten el mismo tipo de distribución, se propone realizar la comparación entre subgrupos de animales del mismo tipo de distribución.

Todos los cálculos necesarios se pueden realizar utilizando el programa desarrollado y presentado en este trabajo, que se puede descargar para su evaluación, gratuitamente y sin ningún tipo de limitación de uso, en la siguiente dirección: www.unavarra.es/rmga/add/.

\section{Bibliografía}

Abadía S, Mendizabal J, Alfonso L (2008). Relationship between depth of individual backfat layers and size of their constituent adipocytes in sows. Acta Agriculturae Scandinavica Section A-Animal Science 58(3): 161-163.

Alfonso L (2004). Subcutaneous fat and loin development in the Basque Black Pied pig breed. Archivos de Zootecnia 53: 415-418.
Alfonso L, Mourot J, Insausti K, Mendizabal JA, Arana A (2005). Comparative description of growth, fat deposition, carcass and meat quality characteristics of Basque and Large White pigs. Animal Research 54: 33-42.

Alzón M, Mendizabal JA, Arana A, Alberti P, Purroy $A$ (2007). Adipocyte cellularity in different adipose depots in bulls of seven Spanish breeds slaughtered at two body weights. Animal 1: 261-267.

Cruz GD, Strathe AB, Rossow HA, Fadel JG (2012). Characterizing bovine adipocyte distribution and its relationship with carcass and meat characteristics using a finite mixture model. Journal of Animal Science 90: 2995-3002.

DeMartinis FD, Francendese A (1982). Very small fat cell populations: mammalian occurrence and effect of age. Journal of Lipid Research 23(8): 1107-20.

Dodson M, Hausman G, Guan L, Du M, Rasmussen T, Poulos S, Mir P, Bergen W, Fernyhough M, McFarland D, Rhoads R, Soret B, Reecy J, Velleman S, Jiang Z (2010). Lipid metabolism, adipocyte depot physiology and utilization of meat animals as experimental models for metabolic research. International Journal of Biological Sciences 6(7): 691-699.

Eliasson B, Smith U, Mullen S, Cushman SW, Sherman AS, Yang J (2014). Amelioration of insulin resistance by rosiglitazone is associated with increased adipose cell size in obese type 2 diabetic patients. Adipocyte 3(4): 1-8.

Fisher NI, Mammen E, Marron JS (1994). Testing for multimodality. Computational Statistics \& Data Analysis 18: 499-512.

Freeman JB, Dale R (2013). Assesing bimodality to detect the presence of a dual cognitive process. Behaviour Research 45: 83-97.

Hadji L, Berger E, Soula H, Vidal H, Géloën A (2014). White adipose tissue resilience to insulin deprivation and replacement. PLOS ONE 9(8): e106214.

Hartigan PM (1985). Algorithm AS 217: Computation of the Dip statistic to test for unimodality. Applied Statistics 34(3): 320-325. 
Hartigan JA, Hartigan PM (1985). The Dip test of unimodality. The Annals of Statistics 13(1): 70-84.

Hood RL, Allen CE (1973). Cellularity of bovine adipose tissue. Journal of Lipid Research 14(6): 605-610.

Jo J, Gavrilova O, Pack S, Jou W, Mullen S, Sumner $A E$, Cushman SW, Periwal V (2009). Hypertrophy and/or Hyperplasia: Dynamics of Adipose Tissue Growth. PLoS Comput Biol 5: e1000324.

Jo J, Shreif Z, Periwal V (2012). Quantitative dynamics of adipose cells. Adipocyte 1(2): 80-88.

Knapp TR (2007). Bimodality Revisited. Journal of Modern Applied Statistical Methods 6(1): article 3.

Maechler M (2013). Diptest: Hartigan's dip test statistic for unimodality - corrected code. R package version 0.75-5. Disponible en http:// CRAN.R-project.org/package $=$ diptest (10 octubre 2014).

McLaughlin T, Sherman A, Tsao P, Gonzalez O, Yee G, Lamendola C, Reaven GM, Cushman SW (2007). Enhanced proportion of small adipose cells in insulin resistant vs. insulin-sensitive obese individuals implicates impaired adipogenesis. Diabetologia 50: 1707-1715.

Mechler F (2002). DIPTST1, the fix for Hartigan's DIPTST algorithm. Disponible en: http://lib.stat. cmu.edu/apstat/217 (10 octubre 2014).

Mendizabal JA, Soret B, Purroy A, Arana A, Horcada A (1997). Influence of sex on cellularity and lipogenic enzymes of Spanish lamb breeds (Lacha and Rasa Aragonesa). Animal Science 64: 283-289.

Mendizabal JA, Thériez M, Bas P, Normand J, Aurousseau B, Purroy A (2004). Fat firmness of subcutaneous adipose tissue in intensively reared lambs. Small Ruminant Research 53: 173-180.
Mersmann HJ, Goodman JR, Brown LJ (1975). Development of swine adipose tissue: morphology and chemical composition. Journal of Lipid Research 16(4): 269-279.

Muratov AL, Gnedin OY (2010). Modeling the metallicity distribution of globular clusters. The Astrophysical Journal 718: 1266.

Pfister R, Schwarz KA, Janczyk M, Dale R, Freeman JB (2013). Good things peak in pairs: a note on the bimodality coefficient. Frontiers in Psychology 4: 700.

SAS Institute Inc. (1989). SAS/STAT User's Guide Version 6, Fourth Edition, Vol. 1, Cary, NC, 943 pp.

Soret B, Mendizabal JA, Arana A, Purroy A, Eguinoa $P$ (1998). Cellularity and lipogenic enzyme activity in Lacha and Rasa Aragonesa lambs during growth. Small Ruminant Research 29: 103-112.

Soula HA, Géloën A, Soulage CO (2015). Model of adipose tissue cellularity dynamics during food restriction. Journal of Theoretical Biology 364: 189-196.

Soula HA, Julienne $H$, Soulage CO, Géloën A (2013). Modelling adipocytes size distribution. Journal of Theoretical Biology 332: 89-95.

Sun K, Kusminski CM, Scherer PE (2011). Adipose tissue remodelling and obesity. The Journal of Clinical Investigation 121: 2094-2101.

Whitehurst GB, Beitz DC, Cianzio D, Topel DG, Johnson DC (1981). Examination of a lognormal distribution equation for describing distributions of diameters of bovine adipocytes. Journal of Animal Science 53: 1236-1245.

(Aceptado para publicación el 23 de junio de 2015) 